\title{
Effects of Higher-Order Laue Zone Reflections on Atomic Resolution TEM Images of Single Layer and Bilayer Graphene
}

\author{
Hwang Su Kim \\ Department of Physics, Kyungsung University, Busan 608-736, Korea: (jwa@ks.ac.kr)
}

In the recent reports [1,2], one can note that the atomic resolution transmission electron microscope (TEM) images of graphene show 3-hold rotational lattice symmetry rather than the 6-fold one, which fold in the [0001] projected potential of a perfect single layer (SLG) or bilayer graphene (BLG). To understand clearly this phenomenon we need first to examine property of the exit wave function of the images in theory. If the free standing sheet of SLG or BLG is assumed to be just a section of the perfect three dimensional graphite crystal, then the Bloch wave formulation of the dynamical theory can be used when higher-order Laue zone reflections explicitly are taken into account [3]. The wave function at the exit surface plane of a graphite object with [0001] orientation can be expressed [4] as:

$$
\Psi_{\mathrm{e}}(\mathbf{X})=\Sigma_{\mathrm{G}}\left(\Sigma_{\mathrm{L}} \phi_{\mathrm{G}+\mathrm{L}}(\mathrm{t}) \cdot \exp [2 \pi \mathrm{i} \mathbf{L} \cdot \mathbf{t}]\right) \cdot \exp [2 \pi \mathrm{i} \mathbf{G} \cdot \mathbf{X}]
$$

where the diffracted electron amplitude given by

$$
\phi_{\mathrm{G}+\mathrm{L}}=\sum_{\mathrm{j}} \alpha^{*(\mathrm{j})} \cdot \mathrm{C}_{\mathrm{G}+\mathrm{L}}{ }^{(\mathrm{j})} \cdot \exp \left(2 \pi \mathrm{i} \gamma^{(\mathrm{j})} \mathrm{t}\right) .
$$

Here $\mathbf{G}=(h k .0)$ and $\mathbf{L}=(00 . l)$ are reciprocal lattice vectors in $(h k .0)$ plane and its perpendicular direction, respectively. $\mathbf{X}$ is a position vector in the plane. $\mathbf{t}$ is the thickness of a graphite object in [0001]direction. $\alpha^{*(\mathrm{j})}$ is the excitation coefficient of Bloch wave of $\mathrm{j}$ in crystal. For the meanings of other symbols in (1), one should refer to [5]. If $\mathrm{C}_{\mathrm{G}+\mathrm{L}}{ }^{(\mathrm{j})} \neq \mathrm{C}-{ }_{\mathrm{G}+\mathrm{L}}{ }^{(j)}$, then $\Psi_{\mathrm{e}}(\mathbf{X}) \neq \Psi_{\mathrm{e}}(-\mathbf{X})$. That is, the wave function has not inverse symmetry. In a perfect graphite crystal with hexagonal $P 6_{3} m c$ (186) structure $(\mathrm{a}=0.246 \mathrm{~nm}, \mathrm{c}=0.671 \mathrm{~nm})$, the structure factor relations: $F(h k . l)=F(-h-k . l)$ for $l=$ even number and $F(h k . l)=-F(-h-k . l)$ for $l=$ odd number fold. This relation leads to $\mathrm{C}_{\mathrm{G}+\mathrm{L}}{ }^{(\mathrm{j})} \neq \mathrm{C}-\mathrm{G}_{\mathrm{G}+\mathrm{L}}{ }^{(\mathrm{j})}$ for $l=$ odd number and then the relation $\Psi_{\mathrm{e}}(\mathbf{X}) \neq \Psi_{\mathrm{e}}(-\mathbf{X})$ is expected, if the amplitudes of higher-order Laue zone reflections is not ignorable. If crystal object thickness is ultra thin, the diffracted amplitudes for zero and higher order Laue reflections are comparable [3]. This result means that the atomic resolution images of SLG/BLG may not have inverse lattice symmetry. That is, the images of SLG/BLG may have 3-fold rotational lattice symmetry rather than the 6-fold one.

In image simulations using (1) to confirm this theoretical prediction, it was assumed that the thickness of SLG is $\mathrm{t}=\mathrm{c} / 2$ from $-\mathrm{c} / 4$ to $\mathrm{c} / 4$ and for BLG $\mathrm{t}=\mathrm{c}$ from $-\mathrm{c} / 4$ to $5 \mathrm{c} / 4$. Fig. $1 \mathrm{a}$ is the phase image obtained from the reconstructed exit wave function using observed 15 lattice images of SLG taken by Berkeley's TEM0.5 [2]. The image clearly has not inverse lattice symmetry. Fig. 1b is the simulated phase image matched with $a$. In fact the phase image of SLG in $b$ was simulated with $l=0 \sim \pm 2$ only. If the full extension of $l(l=0 \sim \pm 8)$ is taken into account, the image show almost inverse lattice symmetry, i.e. 6-fold rotation lattice symmetry. It is thought that this result simply reflects the fact that the electron density on the surface of SLG may not be exactly the same as the density in between atomic plane layers in [0001] direction in graphite crystal. Also the focus spread factor $\Delta_{\mathrm{s}}=2 \mathrm{~nm}$ was given in $b$. This factor can be thought as a remnant after the reconstruction of the exit wave function. It was found the aspect of breaking of the inverse lattice symmetry sensitively depends on $\Delta_{\mathrm{s}}$. In Fig. 2, $a$ and $b$ are the simulated phase image with $\Delta_{\mathrm{s}}=1.5 \mathrm{~nm}$ for SLG and BLG 
respectively. As one can see, the degree of breaking of inverse asymmetry in image of $a$ is less severe than Fig. 1. The images in Fig. 2 are very similar to the cover image in [6]. Again the simulation image in $a$ was give $l=0 \sim \pm 2$ only. But in the case of $b$ for BLG the image shows no significant difference between those with $l=0 \sim \pm 2$ and $l=0 \sim \pm 8$. This means that the case of BLG with double thickness of SLG, the effect of the electron density configuration on the surface already is less important on images than the case of SLG. It should be noted that although the image of BLG in Fig. 2b show generally 6-fold rotational lattice symmetry, many cases of TEM simulated images with a certain spherical aberration $\left(\mathrm{C}_{\mathrm{s}}\right)$ and a defocus $(\Delta \mathrm{f})$ show strong the asymmetry of lattice inversion. In summary even in cases of SLG and BLG regarded as a two dimensional crystal, the conventional Bloch wave formulation can be used in simulations when higher-order Laue reflections are properly taken into account in the theory. However for the case of SLG, the factor of electron density re-configuration on the surface seems to be important to completely understand for the inverse asymmetry on the image as shown in Fig. 2a, and the further investigation for this matter would be interest in future study.

[1] Ç.Ö. Girit et al., Science 323 (2009) 1705-1708.

[2] A. Dato et al., Chem. Commun. issue 40 (2009) 6095-6097.

[3] H.S. Kim, S.S. Sheinin, $51^{\text {st }}$ Annual Meeting of MSA, San Francisco (1993) 450-451.

[4] H.S. Kim, Korean J. Electron Microscopy 37 (2007) 259-269.

[5] P. Hirsch, A. Howie, R.B. Nicholson, D.W. Pashley, and M.J. Whelan, Electron Microscopy of Thin Crystals, Krieger Publ., Huntington (N.Y.) 1977.

[6] J.R. Jinschek et al., Cover image in Microsc. Analys (suppl.) Nov./Dec. (2009) Asia/Pacific

[7] The free release of the image by Berkeley's TEAM0.5 (Fig. 1a) is gratefully acknowledged.
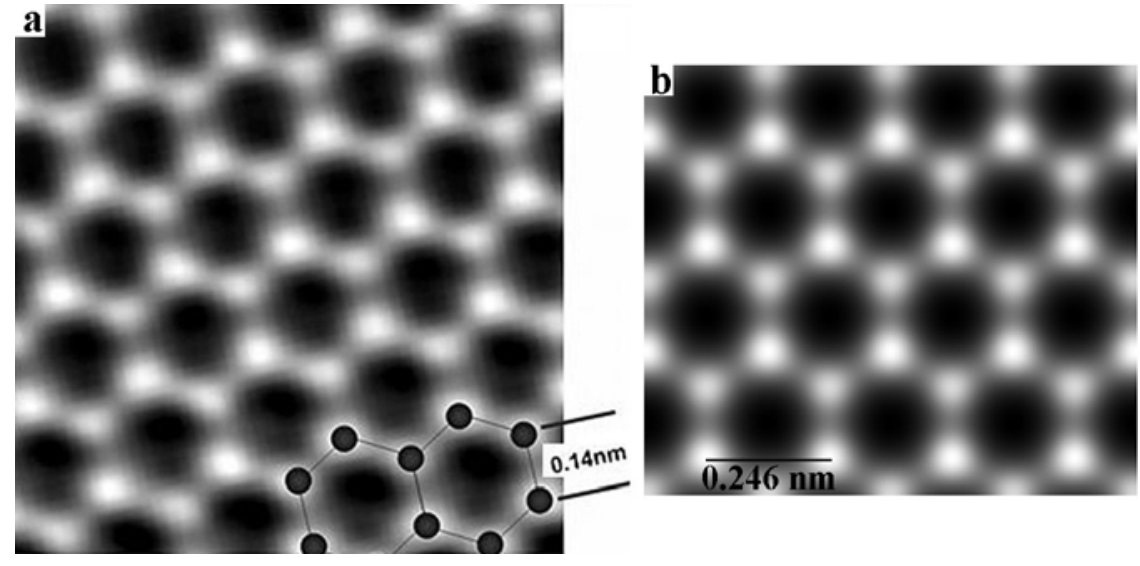

Fig. 1. a: the phase image of reconstructed exit wave function for SLG [2], b: the corresponding simulated phase image for SLG with $\mathrm{C}_{\mathrm{s}}=\Delta \mathrm{f}=0$, the focus spread $\Delta_{\mathrm{s}}=2 \mathrm{~nm}$ and $80 \mathrm{kV}$ accelerating voltage.
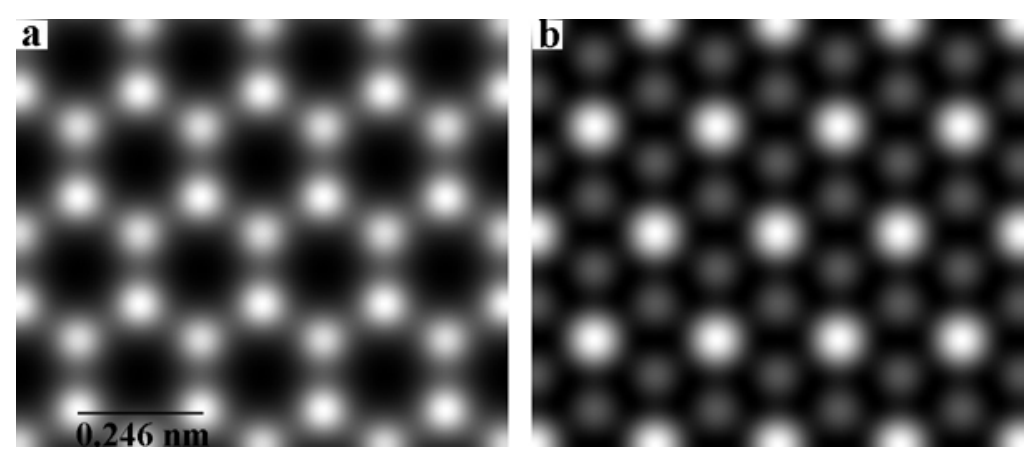

Fig. 2. a: the simulated phase image for SLG [2], b: for BLG. $\mathrm{C}_{\mathrm{s}}=\Delta \mathrm{f}=0$, focus spread $\Delta_{\mathrm{s}}=1.5 \mathrm{~nm}$ and $80 \mathrm{kV}$ accelerating voltage were given in simulations. 